International Journal of Modern Physics B, Vol. 15, Nos. 10 \& 11 (2001) 1385-1388

(C) World Scientific Publishing Company

\title{
HIGH-ORDER COUPLED CLUSTER RESULTS FOR QUANTUM ANTIFERROMAGNETS AND THEIR PHASE TRANSITIONS
}

\author{
R. F. BISHOP AND D. J. J. FARNELL \\ Department of Physics, UMIST, P.O. Box 88, Manchester M60 1QD, UK \\ E-Mail: R.F.Bishop@umist.ac.uk
}

\begin{abstract}
The normal coupled cluster method is implemented to high orders in a systematic approximation scheme, and is shown to give accurate ground- and excited-state properties of anisotropic Heisenberg antiferromagnets and their quantum phase transitions at zero temperature.
\end{abstract}

\section{Introduction}

The coupled cluster method $(\mathrm{CCM})^{1}$ has been applied in recent years with great success to various magnetic lattices (see, for example, Refs. 2-4 and references therein). We have shown ${ }^{2}$ how the systematic incorporation of multispin correlations for quantum spin-lattice systems can be very efficiently implemented within the framework of the CCM. The method is not restricted, for example, to bipartite lattices or to non-frustrated systems, and can thus deal with problems ${ }^{2-4}$ where quantum Monte Carlo (QMC) techniques would be faced with the wellknown "minus-sign problem." We typically find that critical points arise in the solution of the CCM equations as some internal coupling parameter is varied, and we have shown ${ }^{2-4}$ how these are associated with the quantum (zero-temperature) phase transitions between states of different quantum order. We present here new results of high-order CCM calculations for the ground- and excited-state properties of the spin-half anisotropic Heisenberg or $X X Z$ model on the square lattice. In global spin coordinates the $X X Z$ Hamiltonian is specified as follows,

$$
H=\sum_{\langle i, j\rangle}\left[s_{i}^{x} s_{j}^{x}+s_{i}^{y} s_{j}^{y}+\Delta s_{i}^{z} s_{j}^{z}\right]
$$

where the sum on $\langle i, j\rangle$ counts all nearest-neighbour pairs once. On the square lattice the model has no exact solution, but is expected to have a phase transition at about $\Delta=1$ between a phase of Néel-like order in the $z$-direction for $\Delta \gtrsim 1$ and a similar $x-y$ planar phase for $-1<\Delta \lesssim 1$. For $\Delta<-1$ the system is ferromagnetic.

\section{The coupled cluster method}

The exact ket and bra ground states are parametrized independently within the normal coupled cluster method (NCCM) as,

$$
\begin{gathered}
|\Psi\rangle=\mathrm{e}^{S}|\Phi\rangle ; \quad S=\sum_{I \neq 0} \mathcal{S}_{I} C_{I}^{+}, \\
\langle\tilde{\Psi}|=\langle\Phi| \tilde{S} \mathrm{e}^{-S} ; \quad \tilde{S}=1+\sum_{I \neq 0} \tilde{S}_{I} C_{I}^{-},
\end{gathered}
$$


in terms of a suitably chosen normalised reference state $|\Phi\rangle$, with respect to which the set of mutually commuting many-body operators $\left\{C_{I}^{+}\right\}$forms a complete set of creation operators. We define $C_{I}^{-} \equiv\left(C_{I}^{+}\right)^{\dagger}$, and $C_{0}^{+} \equiv 1$, the identity operator. Since $\left\langle\Phi\left|C_{I}^{+}=0=C_{I}^{-}\right| \Phi\right\rangle \forall I \neq 0$, by construction, we note that the normalisation conditions, $\langle\tilde{\Psi} \mid \Psi\rangle=\langle\Phi \mid \Psi\rangle=\langle\Phi \mid \Phi\rangle \equiv 1$, follow immediately.

The ground-state Schrödinger equations,

$$
H|\Psi\rangle=E_{g}|\Psi\rangle ; \quad\langle\tilde{\Psi}| H=E_{g}\langle\tilde{\Psi}|,
$$

are completely equivalent to the variational relation $\delta \bar{H}=0$, where $\bar{H} \equiv\langle\tilde{\Psi}|H| \Psi\rangle$. The ground-state correlation coefficients $\left\{S_{I}, \tilde{S}_{I}\right\}$ are thereby obtained as follows,

$$
\begin{aligned}
& \delta \bar{H} / \delta \tilde{S}_{I}=0 \Rightarrow\left\langle\Phi\left|C_{I}^{-} \mathrm{e}^{-S} H \mathrm{e}^{S}\right| \Phi\right\rangle=0, \quad \forall I \neq 0, \\
& \delta \bar{H} / \delta S_{I}=0 \Rightarrow\left\langle\Phi\left|\tilde{S} \mathrm{e}^{-S}\left[H, C_{I}^{+}\right] \mathrm{e}^{S}\right| \Phi\right\rangle=0, \quad \forall I \neq 0 .
\end{aligned}
$$

Equation (4a) also gives the ground-state energy as,

$$
E_{g}=E_{g}\left(\left\{\mathcal{S}_{I}\right\}\right)=\left\langle\Phi\left|\mathrm{e}^{-S} H \mathrm{e}^{S}\right| \Phi\right\rangle .
$$

In practice, the nested commutator expansions for the similarity transforms in Eqs. $(4 a, b)$ usually terminate at a finite order, and hence the only approximation required is to truncate the otherwise exact (complete) expansions in Eqs. (2a,b) to some subset of the many-body configuration index set $\{I\}$. Formally, Eqs. (4a,b) yield all exact eigenstates for which $\langle\Phi \mid \Psi\rangle \neq 0$.

Excited states $\left|\Psi_{e}\right\rangle$, with $\left\langle\Phi \mid \Psi_{e}\right\rangle=0$, are parametrised in the NCCM as follows,

$$
\left|\Psi_{e}\right\rangle=X^{e} \mathrm{e}^{S}|\Phi\rangle ; \quad X^{e}=\sum_{I \neq 0} X_{I}^{e} C_{I}^{+}
$$

The excited-state Schrödinger equation, $H\left|\Psi_{e}\right\rangle=E_{e}\left|\Psi_{e}\right\rangle$, may be combined with its ground-state counterpart in Eq. (3) to give,

$$
\epsilon_{e} X^{e}|\Phi\rangle=\mathrm{e}^{-S}\left[H, X^{e}\right] \mathrm{e}^{S}|\Phi\rangle ; \quad \epsilon_{e} \equiv E_{e}-E_{g} .
$$

By projecting Eq. (7) with the set of states $\left\{\langle\Phi| C_{I}^{-} ; I \neq 0\right\}$, we obtain a set of linear generalised eigenvalue equations for the excitation coefficients $\left\{X_{I}^{e}\right\}$ as eigenfunctions and the excitation energies $\left\{\epsilon_{e}\right\}$ as eigenvalues.

We turn now to the choice of $|\Phi\rangle$ and the operators $\left\{C_{I}^{+}\right\}$for the case of spin-half quantum antiferromagnets on bipartite lattices, in regimes where the corresponding classical limit is described by a Néel-like order. It is then convenient to introduce a different local quantisation axis and spin coordinates on each sublattice, by a suitable rotation in spin space, so that the corresponding reference state becomes a fully aligned ("ferromagnetic") state, with all spins pointing along, say, the negative $z$-axis in the corresponding local axes. In the same local axes, the configuration indices $I \rightarrow\left\{k_{1}, k_{2}, \cdots, k_{M}\right\}$, a set of site indices, such that $C_{I}^{+} \rightarrow s_{k_{1}}^{+} s_{k_{2}}^{+} \cdots s_{k_{M}}^{+}$, where $s_{k}^{+} \equiv s_{k}^{x}+\mathrm{i} s_{k}^{y}$ is the usual spin-raising operator at site $k$. For the Hamiltonian of Eq. (1) we choose the $z$-aligned Néel state as our reference state (which is the exact ground state for $\Delta \rightarrow \infty$, and is expected to be a good starting point for all $\Delta>1$, down to the expected phase transition at $\Delta=1$ ). We then perform a rotation of the up-pointing spins by $180^{\circ}$ about the $y$-axis, such that $s^{x} \rightarrow$ 
$-s^{x}, s^{y} \rightarrow s^{y}, s^{z} \rightarrow-s^{z}$. The Hamiltonian of Eq. (1) may thus be written in these local coordinates as,

$$
H=-\frac{1}{2} \sum_{\langle i, j\rangle}\left[s_{i}^{+} s_{j}^{+}+s_{i}^{-} s_{j}^{-}+2 \Delta s_{i}^{z} s_{j}^{z}\right] .
$$

The results presented below are based on a localised NCCM approximation scheme, known as the LSUB $m$ scheme, in which we include all multispin correlations over all locales (or "lattice animals") on the lattice defined by $m$ or fewer contiguous sites. We include all fundamental configurations, $I \rightarrow\left\{k_{1}, k_{2}, \cdots, k_{n}\right\}$, with $n \leq m$, which are distinct under the point and space group symmetries of both the lattice and the Hamiltonian. The numbers, $N_{F}$ and $N_{F_{e}}$, of such fundamental configurations for the ground and excited states, respectively, may be further restricted by the use of additional conservation laws. For example, the Hamiltonian of Eq. (1) commutes with the total uniform magnetisation, $s_{T}^{z}=\sum_{k} s_{k}^{z}$, where the sum on $k$ runs over all lattice sites. The ground state lies in the $s_{T}^{z}=0$ subspace, and hence we exclude configurations with an odd number of spins or with unequal numbers of spins on the two equivalent sublattices. Similarly for the excited states, since we are only interested in the lowest-lying excitation, we restrict the configurations to those with $s_{T}^{z}= \pm 1$, i.e., to single-magnon spin-wave-like excitations.

\section{Results}

We present typical LSUB $m$ results (with $m=2,4,6,8$ ) for the energy per spin $\left(E_{g} / N\right)$ and the sublattice magnetisation $\left(M \equiv-2\left\langle\tilde{\Psi}\left|s_{k}^{z}\right| \Psi\right\rangle\right.$ in the local rotated spin coordinates) of the ground state, and the gap $\left(\epsilon_{e}\right)$ of the lowest-lying excited state, for the $X X Z$ model on the square lattice. We find that for $m>2$ the physical branch of ground-state solutions (i.e., the one which becomes exact in the $\Delta \rightarrow \infty$ limit) terminates at a critical value $\Delta_{c}$, such that for $\Delta<\Delta_{c}$ no real solution exists. This is taken to be a signal of the expected phase transition at (or near) $\Delta=1$. The LSUB $m$ results are compared in Table 1 for the isotropic $(\Delta=1)$ case with results from linear spin-wave theory (LSWT), series expansion techniques, ${ }^{5}$ and quantum Monte Carlo (QMC) simulations. ${ }^{6}$ We also show the results of two simple $m \rightarrow \infty$ extrapolation schemes using the LSUB $m$ results with $m=4,6,8$. Results denoted as "extrapolated(1)" are fitted separately for each quantity to a leading power-law dependence of the form, $y_{m}=a+b m^{-\nu}$; while those denoted as "extrapolated(2)" are fitted either (for case $l=0$ ) to a simple quadratic form, $y_{m}=a_{0}+a_{1} / m+a_{2} / m^{2}$, or (for case $l=1$ ) to a simple Padé form, $y_{m}=\left(a_{0}+a_{1} / m\right)\left(1+b_{1} / m\right)^{-1}$. In Fig. 1 we show the results for $\epsilon_{e}$ and $M$ as functions of $\Delta$, the anisotropy parameter.

\section{Conclusions and outlook}

It is evident from the results presented, and similar results on the linear chain and the cubic lattice, that the CCM can provide very accurate and systematically improvable estimates for the ground- and excited-state properties of quantum magnets. The method deals from the outset with infinite lattices, and hence no finite-size scaling needs to be performed, as in the case of QMC calculations, for 
Table 1. CCM results for the spin-half square-lattice Heisenberg antiferromagnet compared to results of other methods.

\begin{tabular}{lcccccc}
\hline \hline Method & $N_{F}$ & $N_{F_{e}}$ & $E_{g} / N$ & $M$ & $\epsilon_{e}$ & $\Delta_{c}$ \\
\hline LSUB2 & 1 & 1 & -0.64833 & 0.841 & 1.407 & - \\
LSUB4 & 7 & 6 & -0.66366 & 0.765 & 0.852 & 0.577 \\
LSUB6 & 75 & 91 & -0.66700 & 0.727 & 0.610 & 0.763 \\
LSUB8 & 1273 & 2011 & -0.66817 & 0.705 & 0.473 & 0.843 \\
Extrapolated(1) CCM & - & - & -0.66970 & 0.557 & -0.191 & 1.001 \\
Extrapolated(2) $(l=0)$ & - & - & -0.66971 & 0.623 & -0.001 & 1.031 \\
Extrapolated(2) $(l=1)$ & - & - & -0.67062 & 0.616 & -0.020 & 1.044 \\
LSWT & - & - & -0.658 & 0.606 & 0.0 & 1.0 \\
Series Expansions [Ref. 5] & - & - & $-0.6693(1)$ & $0.614(2)$ & - & - \\
QMC [Ref. 6] & - & - & $-0.669437(5)$ & $0.6140(6)$ & - & - \\
\hline \hline
\end{tabular}
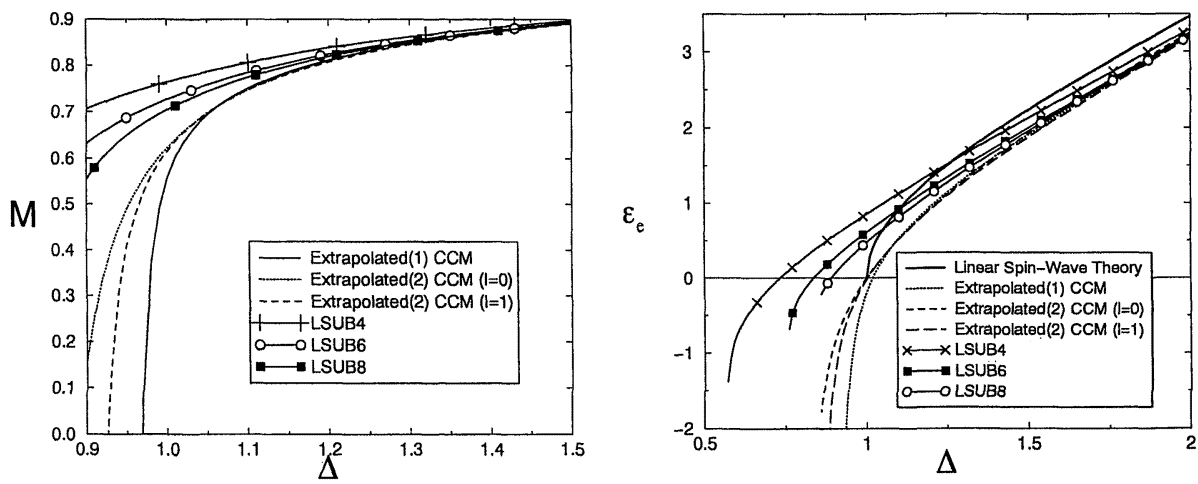

Figure 1. CCM results for the staggered magnetisation and the lowest-lying excitation energy gap for the spin-half square-lattice $X X Z$ model.

example. However, we have shown that we may improve our results further by employing heuristic extrapolation schemes in the LSUB $m$ truncation index, $m \rightarrow \infty$. It will be of great interest to try to study the scaling in this index at a more formal level, and to extend the methodology to deal with systems at finite temperatures.

\section{References}

1. R. F. Bishop, in Microscopic Quantum Many-Body Theories and Their Applications, eds. J. Navarro and A. Polls, Lecture Notes in Physics Vol. 510 (Springer-Verlag, Berlin, 1998), p. 1.

2. C. Zeng, D. J. J. Farnell, and R. F. Bishop, J. Stat. Phys. 90, 327 (1998).

3. R. F. Bishop, D. J. J. Farnell, and J. B. Parkinson, Phys. Rev. B 58, 6394 (1998).

4. R. F. Bishop, D. J. J. Farnell, and C. Zeng, Phys. Rev. B 59, 1000 (1999).

5. Zheng Weihong, J. Oitmaa, and C. J. Hamer, Phys. Rev. B 43, 8321 (1991).

6. A. W. Sandvik, Phys. Rev. B 56, 11678 (1997). 